\title{
Early and continuing education: a prescription for achieving patient-centred care
}

$\mathrm{T}$ hat health care ought to be patient-centred - and that patients want this — seems self-evident. ${ }^{1}$ Patient-centred care has many definitions, but in general, it refers to care that explicitly elicits and addresses patients' perspectives on their health. ${ }^{2}$ Evidence reveals that patients are more satisfied, report more positive experiences and perceive greater improvements in their symptoms in patient-centred models of care..$^{1,3}$

However, some studies have shown inconsistent effects on physiologic outcomes ${ }^{3}$ or worse health-related outcomes with patient-centred models. ${ }^{4}$ Studies to date have not shown decreases in clinical events or in the use of health resources such as emergency department visits. It thus seems likely that bringing the patient's perspective into the system isn't enough to achieve the health outcomes people want and expect.

Even a perfect system won't work if patients don't know how to access it effectively. No matter what resources are available, lack of knowledge about what resources exist both within and outside the health care system - and how to access them keeps people from receiving what they need when they need it. Also, our failure to help patients adequately understand their disease and its treatment underlies much of the suboptimal adherence to treatment that leads to otherwise preventable outcomes. Ironically, health knowledge is more widely available to people than ever before as a result of our information-centred, and Internet and social media-driven culture. Unfortunately, the average person lacks the guidance necessary to be able to leverage this knowledge effectively.

Patient-centred care requires putting the patient, not the system, at the centre. We must stop seeing the health care system as the sole and sufficient mechanism for improving health. Instead, we must empower people to become the solution to achieving their own optimal health by enabling them to connect effectively not only to the system but also to other resources around them, including family and community. What people need most is navigation through all these elements and education about how to engage them effectively.

Once we recognize that the main challenge is education, the solution should be obvious: society should use the mechanisms it already uses to impart core knowledge. First and foremost, this means starting as early as possible — in schools. Schools already provide education about basic aspects of public health such as nutrition, physical fitness and sex education. Why does it stop there? Why not create a mandatory course in how to be a health consumer as part of every standard high school curriculum? The life skills and knowledge involved are as important as anything else we regard as essential for everyone to learn before adulthood.

This should be supplemented by developing and promoting continuing education programs directed at meeting specific needs through key health-related life stages: pregnancy, par- enthood, retirement and aging, and caring for someone else. Such programs should reach out especially to groups most often marginalized, such as those who have low socioeconomic status, face linguistic or cultural barriers, or have been disadvantaged historically (such as aboriginal communities). It is no accident that many of these factors are associated with the worst health outcomes. Health professionals and policy-makers need to partner with community organizations as well as mass media to enhance effectiveness of delivery and ensure appropriateness of content.

Health care providers and policy-makers have also been too passive in response to the arrival of Internet-based technologies and social media, leaving the system far behind much of the population and struggling to catch up. We urgently need to adapt these technologies to help patients navigate through the existing sea of unreliable and often conflicting information.

Future efforts at health reform must emphasize improving the delivery of health, not just the delivery of health care. Although building new physical structures and better systems for care delivery is undeniably important, we have failed to make a commensurate investment in building adequate mechanisms for delivering education about health and health resources to the average citizen. We will likely never see meaningful decreases in hospital and emergency department crowding, nor achieve the health goals of which society ought to be capable, until we adopt a more holistic — and truly patient-centred — approach to delivering health.

Matthew B. Stanbrook MD PhD, Diane Kelsall MD MEd, Noni E. MacDonald MD MSc, Daniel Rosenfield MD, Ken Flegel MDCM MSc, Paul C. Hébert MD MHSc

\section{References}

1. Little P, Everitt H, Williamson I, et al. Preferences of patients for patient centred approach to consultation in primary care: observational study. BMJ 2001;322:468-72.

2. Levenstein JH, McCracken EC, McWhinney IR, et al. The patient-centred clinica method. 1. A model for the doctor-patient interaction in family medicine. Fam Pract 1986;3:24-30.

3. Griffin SJ, Kinmonth A-L, Veltman MW, et al. Effect on health-related outcomes of interventions to alter the interaction between patients and practitioners: a systematic review of trials. Ann Fam Med 2004;2:595-608.

4. Kinmonth A-L, Woodcock A, Griffin S, et al; the Diabetes Care From Diagnosis Research Team. Randomised controlled trial of patient centred care of diabetes in general practice: impact on current wellbeing and future disease. BMJ 1998;317:1202-8.

Competing interests: See www.cmaj.ca/site/misc/cmaj_staff.xhtml. None declared for Paul Hébert.

Affiliations: See www.cmaj.ca/site/misc/cmaj_staff.xhtml. Daniel Rosenfield is with the Faculty of Medicine, University of Toronto and the Department of Paediatrics, Hospital for Sick Children, Toronto, Ont. Paul Hébert is with the Ottawa Health Research Institute, Ottawa, Ont.

Correspondence to: $C M A J$ editor, pubs@cmaj.ca

CMAJ 2012. DOI:10.1503/cmaj.111687 